\title{
Internet das coisas e aprendizado de máquina na área da saúde: uma análise bibliométrica da produção científica de 2009 a 2019
}

\author{
Internet of things and machine learning in the health field: a bibliometric \\ analysis of scientific production from 2009 to 2019
}

Internet de las cosas y el aprendizaje automático en el área de la salud: un análisis bibliométrico de la producción científica de 2009 hasta 2019

Paulo Sergio da Conceição Moreira ${ }^{1, a}$

paulomoreira@ufpr.br | https://orcid.org/oooo-0002-3168-2177

Byanca Neumann Salerno ${ }^{1, a}$

byancasalerno@ufpr.br | https://orcid.org/0000-0002-1609-4684

Denise Fukumi Tsunoda ${ }^{1, b}$

dtsunoda@ufpr.br | https://orcid.org/o0oo-0002-5663-4534

\footnotetext{
${ }^{1}$ Universidade Federal do Paraná, Programa de Pós-Graduação em Gestão da Informação. Curitiba, PR, Brasil.

Mestrado em Gestão da Informação pela Universidade Federal do Paraná.

${ }^{\mathrm{b}}$ Doutorado em Engenharia Elétrica e Informática Industrial - Engenharia Biomédica pela Universidade Tecnológica Federal do Paraná.
}

\section{Resumo}

A internet das coisas e o aprendizado de máquina são temas emergentes na área da saúde com potencial para otimizar a área e criar um sistema de saúde inteligente em virtude do envelhecimento da população. Este artigo analisa a produção científica do período de 2009 a 2019 a respeito da internet das coisas e do aprendizado de máquina na área da saúde. Utiliza metodologia bibliométrica em 1.353 artigos recuperados na base de dados Web of Science. Constata um crescimento da produção científica sobre o tema, sendo os Estados Unidos o principal polo de pesquisa na área. Identifica os autores mais produtivos e com maior impacto, periódicos mais produtivos, colaboração entre países e palavras-chave utilizadas, bem como suas relações. Incentiva que novas pesquisas explorem as abordagens identificadas no estudo.

Palavras-chave: Internet das coisas; Aprendizado de máquina; Bibliometria; Saúde; Tecnologia. 


\section{Abstract}

The internet of things and machine learning are emerging issues with the potential to optimize the health field and create an intelligent health system due to the aging population. This article analyzes the scientific production of the period from 2009 to 2019 regarding the internet of things and machine learning in the health area. It uses bibliometric methodology in 1.353 articles retrieved from the Web of Science database. It notes an increase in scientific production on the subject, the United States being the main research center in this area. It identifies the most productive and influential authors, the most productive journals, collaboration between countries and keywords used, as well as their relations. It encourages new research to explore the approaches identified in the study.

Keywords: Internet of things; Machine learning; Bibliometrics; Health; Technology.

\section{Resumen}

La internet de las cosas y el aprendizaje de máquinas son temas emergentes en el área de la salud con potencial para optimizar el área y crear un sistema de salud inteligente en virtud del envejecimiento de la población. Este artículo analiza la producción científica del período de 2009 hasta 2019 respecto a internet de las cosas y del aprendizaje de máquina en el área de la salud. Utiliza metodología bibliométrica en 1.353 artículos recuperados en la base de datos Web of Science. Constata un crecimiento de la producción científica sobre el tema, siendo los Estados Unidos el principal polo de investigación en el área. Identifica a los autores más productivos y con mayor impacto, periódicos más productivos, colaboración entre países y palabras clave utilizadas, así como sus relaciones. Estimula a que nuevas investigaciones exploren los enfoques identificados en el estudio.

Palabras clave: Internet de las cosas; Aprendizaje de máquinas; Bibliometría; Salud; Tecnología.

\footnotetext{
Contribuição dos autores:

Concepção e desenho do estudo: Paulo Sergio da Conceição Moreira, Byanca Neumann Salerno e Denise Fukumi Tsunoda. Redação do manuscrito: Paulo Sergio da Conceição Moreira, Byanca Neumann Salerno e Denise Fukumi Tsunoda.

Declaração de conflito de interesses: não há.

Fontes de financiamento: Coordenação de Aperfeiçoamento de Pessoal de Nível Superior (Capes).

Considerações éticas: não há.
} Aquisição, análise ou interpretação dos dados: Paulo Sergio da Conceição Moreira, Byanca Neumann Salerno e Denise Fukumi Tsunoda.

Revisão crítica do conteúdo intelectual: Paulo Sergio da Conceição Moreira, Byanca Neumann Salerno e Denise Fukumi Tsunoda.

Agradecimentos/Contribuições adicionais: não há.

Histórico do artigo: submetido: 23 fev. 2019 | aceito: 13 out. 2019 | publicado: 31 mar. 2020.

Apresentação anterior: não houve.

Licença CC BY-NC atribuição não comercial. Com essa licença é permitido acessar, baixar (download), copiar, imprimir, compartilhar, reutilizar e distribuir os artigos, desde que para uso não comercial e com a citação da fonte, conferindo os devidos créditos de autoria e menção à Reciis. Nesses casos, nenhuma permissão é necessária por parte dos autores ou dos editores. 


\section{Introdução}

Devido ao desenvolvimento tecnológico, verifica-se uma grande quantidade de dados disponíveis no setor da saúde, oriundos de fontes como sensores vestíveis, rastreadores de atividade, dispositivos de monitoramento contínuo e dispositivos médicos autônomos ${ }^{1-2}$. Contudo, muitas vezes, esses dados são armazenados em planilhas eletrônicas, por exemplo, e não fazem parte dos registros eletrônicos de saúde².

Visando à solução desse problema, a internet das coisas (do inglês Internet of Things - IoT) surge como uma das principais áreas-chave da tecnologia e vem recebendo uma grande atenção nos últimos anos ${ }^{3}$, uma vez que pode atuar como o elo entre diferentes fontes de dados e os dispositivos médicos utilizados, possibilitando diagnósticos mais ágeis e precisos².

A internet das coisas surge como uma tecnologia na qual suas aplicações auxiliam diferentes tipos de negócios, como a agricultura, o transporte e a saúde ${ }^{4}$. Na área da saúde, graças ao desenvolvimento de sensores, há possibilidade de atuação em um ambiente de aprendizagem inteligente, criando oportunidades para a otimização da área ${ }^{5}$.

Muito mais do que um elemento de conexão entre dados e dispositivos médicos, a exploração da internet das coisas na área da saúde faz-se necessária devido a fatores como o envelhecimento da população e o aumento das condições crônicas de saúde, que demandam que os indivíduos estejam aptos a cuidar da sua saúde no seu próprio lar ${ }^{4,6}$. Tal mudança de comportamento surge por conta do estresse que os ambientes hospitalares podem provocar, principalmente em idosos e crianças ${ }^{4}$.

Neste cenário, outra tecnologia, que somada à internet das coisas, pode proporcionar o desenvolvimento de um sistema de saúde inteligente consiste no aprendizado de máquina (em inglês, machine learning), cujo uso na área da saúde apresenta potencial para reduzir custos de tratamento ${ }^{1,7}$, posto que é possível desenvolver um conjunto de ferramentas de alerta e gerenciamento de riscos, que vão desde a predição de possíveis surtos de epidemias à geração de resultados de forma ágil ${ }^{1}$.

Um sistema de saúde inteligente pode ser implementado em todos os níveis, desde o monitoramento da temperatura dos bebês até o rastreamento dos sinais vitais nos idosos ${ }^{4}$. Entretanto, é preciso a integração de dispositivos individuais com os atuais dispositivos e sistemas utilizados por médicos e profissionais da área da saúde. Para isso, faz-se necessário que os profissionais da saúde utilizem tecnologias como big data, aprendizado de máquina, computação em nuvem e inteligência artificial ${ }^{4}$.

Ademais, cabe ressaltar que, para estas tecnologias serem eficazes na detecção e no gerenciamento de condições de saúde, é preciso que haja dados clínicos significativos, que também sejam acessíveis para os seus usuários domésticos ${ }^{6}$.

Mediante esse contexto, o objetivo do presente artigo consiste em analisar a produção científica do período de 2009 a 2019 a respeito da internet das coisas e do aprendizado de máquina na área da saúde por meio da bibliometria.

\section{Fundamentos básicos de bibliometria}

No século XXI, a informação é disseminada em grande quantidade e velocidade, o que dificulta seu acompanhamento integral por parte dos pesquisadores ${ }^{8}$. Assim, é preciso filtrar e selecionar informações relevantes em meio às disponíveis nas bases de dados. Nesse contexto, os estudos bibliométricos mostramse fontes de informação para diferentes áreas de pesquisa, sendo importantes para subsidiar a evolução tecnológica e científica ${ }^{9}$ pela sua característica de mapear a produção científica ${ }^{8,10}$.

A bibliometria é uma área da ciência da informação com papel importante para avaliar a produção científica, pois seus indicadores podem mostrar a evolução de um campo do conhecimento. Ela foi 
popularizada em 1969, apesar de ter tido origem em 1934 com a denominação original de "bibliografia estatística" e é utilizada em pesquisas sobre a produção científica e disseminação do conhecimento8 .

De natureza quantitativa, essa técnica utiliza a matemática e a estatística para atingir sua finalidade e passou por mudanças desde a sua origem. No início, destinava-se ao estudo de bibliotecas, com foco em identificar estatísticas referentes à quantidade de exemplares disponíveis e gestão do espaço físico, por exemplo. Com o passar do tempo, principalmente devido às inovações digitais e ao fomento da pesquisa pelas instituições e políticas públicas, essa técnica evoluiu e incluiu em suas análises os artigos de periódicos, produtividade de autores e estudo de citações ${ }^{11,12}$.

Entre os principais teóricos que fundamentam a área, estão: Lotka ${ }^{13}$, que aborda a produtividade dos autores; Bradford ${ }^{14}$, que investiga a dispersão do conhecimento em periódicos; e Zipf ${ }^{15}$, que examina a distribuição e frequência das palavras em textos ${ }^{11,16}$. A lei de Lotka, também conhecida como lei dos quadrados inversos, exprime que muitos autores publicam apenas um artigo e poucos autores publicam muitos artigos ${ }^{11,13,17}$. Isso é analisado a partir da distribuição de frequência dos autores e sua produtividade ${ }^{18}$. A expressão matemática é dada por:

$$
y_{x}=c \times 1 / x^{2}
$$

Nesta equação, $y_{x}$ é a quantidade de autores $\operatorname{com}^{x}$ artigos, ${ }^{c}$ é a quantidade de autores com uma única publicação e ${ }^{x}$ é a quantidade de publicações ${ }^{13,17}$. Portanto, com essa fórmula é possível prever quantos autores produziriam ${ }^{x}$ artigos e, de acordo com ela, 25\% das contribuições vão corresponder a $75 \%$ dos autores menos produtivos, enquanto os autores mais produtivos vão representar $50 \%$ das contribuições. Além disso, a aplicação da lei de Lotka depende do período escolhido para análise, pois é preciso considerar um intervalo suficiente para que os autores tenham oportunidade de publicar mais de um ou dois artigos. Esse período geralmente é a partir de $10 \operatorname{anos}^{17}$.

A lei de Bradford, também conhecida como lei de dispersão, analisa a dispersão de assuntos específicos em periódicos de outras áreas. Bradford verificou que existe uma relação inversa entre a quantidade de artigos publicados em uma área e a quantidade de periódicos em que os artigos aparecem ${ }^{14,17}$. Considerando uma área do conhecimento específica, pode-se dizer que um pequeno grupo de periódicos concentra a maior parte das publicações dessa área, enquanto um grupo maior de periódicos contém poucos artigos da área ${ }^{11,17}$. É possível verificar essa premissa separando os periódicos em três zonas, cada qual com a mesma quantidade de artigos, representando cerca de $1 / 3$ da produção total ${ }^{11}$, sendo que a quantidade de periódicos pode variar de uma para outra ${ }^{17}$.

Por sua vez, a lei deZipf, também denominada lei do menor esforço, faz análise da distribuição e frequência de palavras por meio de um ranking de ocorrências, que está diretamente relacionado à importância dos termos dentro de um texto ${ }^{19}$. A referência ao mínimo esforço deve-se ao fato de que Zipf identificou que palavras que exigem pouco esforço para sua utilização são frequentemente utilizadas em textos ${ }^{20}$.

Zipf propôs duas leis acerca da sua teoria: a primeira (fórmula 2) relacionada às palavras de alta frequência e a segunda (fórmula 3) relacionada às de baixa frequência ${ }^{21}$. A primeira lei é formulada pelo produto da ordem de série de uma palavra $(r)$ multiplicado pela frequência $(f)$. Zipf identificou que esse produto equivale a uma constante ${ }^{C}$ ). A fórmula é dada por:

$$
r \times f=c
$$


Já a segunda lei também propõe que as palavras de baixa frequência têm a mesma frequência. Essa lei foi modificada por Boothii e é representada pela seguinte fórmula:

$$
I_{n}=\frac{2 I_{1}}{n(n+1)}
$$

Neste modelo, ${ }^{I n}$ é a quantidade de palavras com frequência $n, \mathrm{e}^{I_{1}}$ é a quantidade de palavras com frequência 1. Entretanto, pelo fato de cada uma dessas leis referir-se a uma extremidade (alta e baixa frequência, respectivamente), Goffman ${ }^{\mathrm{ii}}$ adaptou a teoria, propondo um ponto de transição entre as duas leis, denominado Ponto de transição de Goffman ${ }^{T}$ ).

Na região de transição estão as palavras com maior carga semântica e, portanto, mais adequadas à representação temática de um texto ${ }^{21}$. A equação 4 representa essa teoria:

$$
n=\frac{-1+\sqrt{1+8 I_{1}}}{2}
$$

Por ser adaptada da segunda lei de Zipf, o elemento ${ }^{n}$ representa o ponto de transição $^{T}$ de Goffman e $I_{1}$ a quantidade de palavras com frequência 1 no ranking. Na seção seguinte, são abordados exemplos de estudos da área da saúde que utilizam a teoria bibliométrica apresentada.

Outra métrica usualmente utilizada em pesquisas bibliométricas relacionadas a citações é o índice-h, também denominado h-index. Esse índice mensura o impacto dos pesquisadores a partir dos seus artigos mais citados. Basicamente, se um autor possui índice-h de 25, então ele tem 25 artigos com pelo menos 25 citações. Para identificar o índice-h de um autor, basta ordenar seus artigos em quantidade decrescente de citações. A posição do h artigo, com pelo menos h citações, é o índice-h do autor ${ }^{17}$.

\section{A bibliometria na área da saúde}

A bibliometria vem sendo aplicada em diversos estudos na área da saúde no âmbito nacional e internacional. Soteriades e Falagas ${ }^{22}$ realizam uma análise bibliométrica nas áreas de medicina preventiva, medicina ocupacional/ambiental, epidemiologia e saúde pública. Para isso, utilizam a base de dados Journal Citation Reports (JCR) no período de 1995 a 2003. Os autores avaliam a produção científica dentro desses temas por países.

Santin, Nunez e Moura $^{23}$ utilizam a bibliometria para verificar a produção científica brasileira sobre células-tronco de 2000 a 2013 na base Web of Science. Os indicadores analisados na pesquisa são as instituições mais produtivas, as áreas de destaque e a colaboração internacional. Outros autore ${ }^{24}$ examinam a colaboração acadêmica entre profissionais de saúde bucal e pré-natal. Para isso, utilizam a análise de redes sociais para descobrir padrões de comunicação entre os profissionais a partir de periódicos científicos. Os registros foram recuperados da Web of Science e foram utilizados indicadores como citações entre periódicos.

Martins $^{25}$ analisa a produção científica sobre o Zika vírus desde 1940 em bases internacionais por meio da bibliometria e identifica a falta de estudos nacionais sobre o tema. Entre os indicadores utilizados pelo autor estão: quantidade de publicações por ano, quantidade de publicações por periódico e palavras-chave.

Outros autores ${ }^{26}$ analisam a produção científica sobre laringectomia total por meio da bibliometria feita com base em documentos da base Scopus. Para isso, utilizam as leis de Bradford, Lotka e Zipf e outros indicadores de colaboração e produtividade. Há, ainda, pesquisa que analisa a produção científica

\footnotetext{
i Booth AD. A "law" of occurrences for words of low frequency. Inform Control. 1967 Apr.;10(4):386-93.

ii Goffman, W. Mathematical approach to the spread of scientifics ideas: the history of mast cell research. Nature, [s.1], v. 212, p.449-452, Oct. 1966.
} 
brasileira sobre nanotecnologia e saúde na base Web of Science no período de 1995 a 2014, utilizando a ferramenta VantagePoint. Os autores identificam a evolução histórica do tema, as principais instituições que o pesquisam e a colaboração internacional ${ }^{27}$.

Outra pesquisa analisa a produção científica no campo da tecnologia móvel aplicada à saúde de 2006 a 2016, utilizando dados da Scopus. Indicadores como publicações por ano, índice-h, palavras-chave, colaboração entre autores, produtividade entre países e entre instituições são alguns exemplos utilizados ${ }^{28}$.

Outros autores verificam a produção científica sobre neoplasia bucal e reabilitação bucal entre os anos de 1990 a 2016 na base de dados Scopus e identificam a dispersão das publicações e padrões de autoria e citações com base nas leis de Lotka e Bradford ${ }^{29}$. Sweileh ${ }^{30}$, por sua vez, investiga a evolução da literatura sobre desastres naturais na área da saúde a partir de dados da base Scopus de 1900 a 2017. Nesse estudo o autor analisa as publicações por ano, citações recebidas pelos documentos, índice-h, ocorrência de palavraschave, contribuição dos autores e respectivos países e periódicos com maior quantidade de publicações.

\section{Metodologia}

Consiste em um estudo bibliométrico por meio da análise da produção científica que envolve o uso da internet das coisas e do aprendizado de máquina na área da saúde. A coleta dos dados deste estudo ocorreu no dia o5 de abril de 2019 por meio de uma busca na base de dados Web of Science pela expressão ("machine learning OR internet of things") AND "health".

Essa base foi escolhida por cobrir vários campos de estudo e abranger dados desde $1900^{31}$. Foram considerados os campos título, resumo, palavras-chave do autor e keywords plus na base, o acesso se deu via Portal de Periódicos da Capes e o escopo foi limitado a artigos científicos de acesso aberto. O período temporal coletado foi 2009-2019, um intervalo de 10 anos e 4 meses, visto que o ano de 2019 abrangeu pesquisas publicadas até abril, período da coleta de dados. O total de registros recuperados correspondeu a 1.353 trabalhos. Os procedimentos metodológicos adotados neste estudo são elencados no Quadro 1.

Quadro 1 - Procedimentos metodológicos

\begin{tabular}{|c|c|}
\hline Recurso & Análise \\
\hline \multirow{4}{*}{ Autor } & Artigos por ano $22,24-27$ \\
\hline & Índice-h25-27,29 \\
\hline & Número de citações ${ }^{25-27}$ \\
\hline & Lei de Lotka ${ }^{13,23,26}$ \\
\hline \multirow{4}{*}{ Fonte } & Lei de Bradford 20,23,26 \\
\hline & Periódicos $21,22,26,27$ \\
\hline & Instituiç̧ões ${ }^{20,24,25}$ \\
\hline & Colaboração entre países ${ }^{19,20,24,25,27}$ \\
\hline \multirow{2}{*}{ Área } & Palavras-chave 25,27 \\
\hline & Lei de Zipf ${ }^{19-21}$ \\
\hline
\end{tabular}

Fonte: Os autores (2019).

Para a realização da etapa de análise bibliométrica foi utilizada a interface gráfica do pacote desenvolvido em linguagem RBibliometrix10 denominada Biblioshiny, e a ferramenta Microsoft Excel $2017^{\circledR}$. Os resultados obtidos são apresentados na sequência. 


\section{Resultados e discussão}

A primeira análise destinou-se a levantar a quantidade de trabalhos por ano, bem como identificar em termos percentuais quanto cada ano representa para a produção científica do tema do presente estudo. Os resultados são apresentados no Gráfico 1.

Gráfico 1 - Quantidade de registros ao longo dos anos

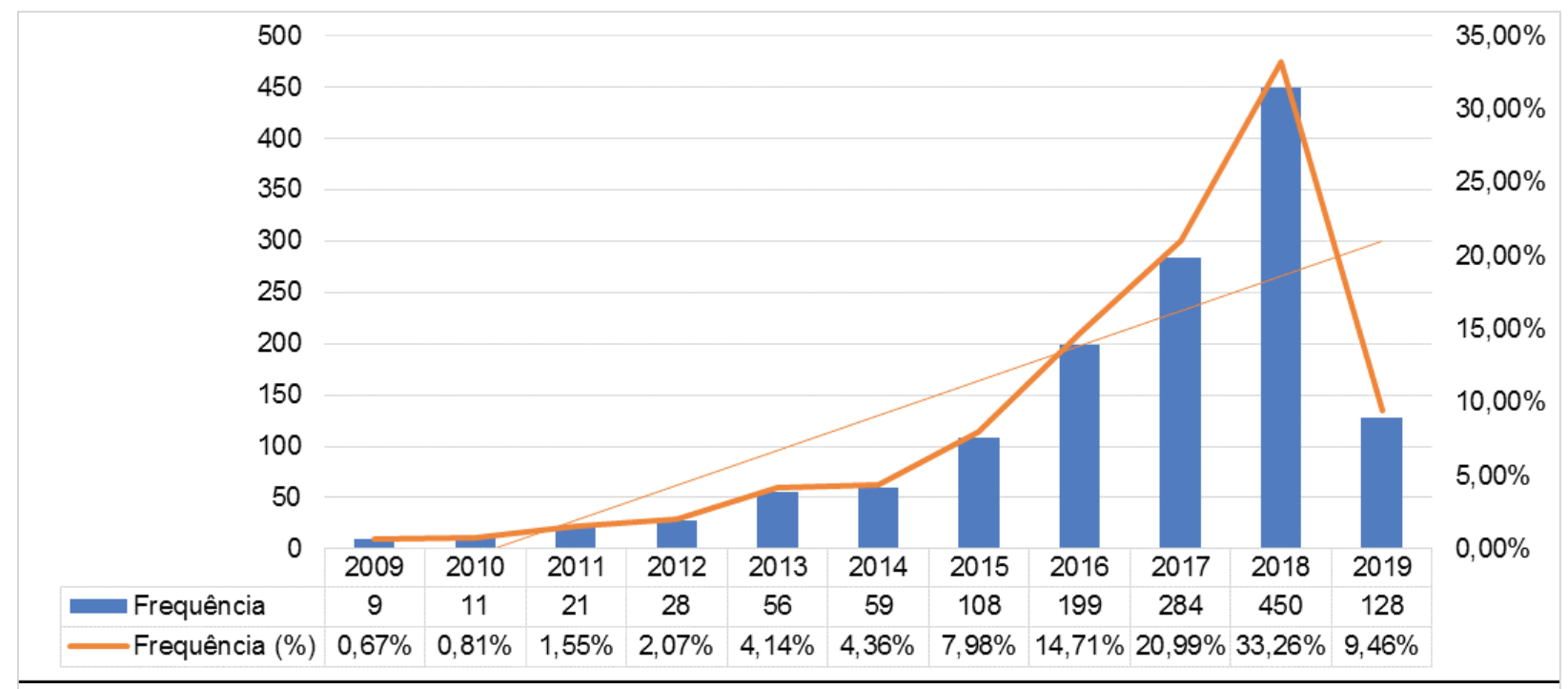

Fonte: Os autores (2019).

Conforme o Gráfico 1, há um crescimento expressivo do volume da produção científica ao longo dos anos, com a produção máxima alcançada no ano de 2018 - 450 artigos representando 33,26\% da produção. Contudo, pelo comportamento da produção científica, esse número tende a ser superado, haja vista que até abril de 2019, o total de artigos produzidos totaliza 128 registros, que correspondem a 9,46\% do volume produzido. No que tange aos autores, 6.745 autores já produziram, pelo menos, um trabalho com a temática abordada neste estudo, dos quais os dez mais produtivos são apresentados na Tabela 1.

Tabela 1 - Autores mais produtivos

\begin{tabular}{lcc}
\multicolumn{1}{c}{ Autor } & Quantidade de artigos & Quantidade de artigos (\%) \\
Zhang Y & 12 & $0,89 \%$ \\
Wang X & 11 & $0,81 \%$ \\
Wang Y & 11 & $0,81 \%$ \\
Cook DJ & 10 & $0,74 \%$ \\
Kim J & 10 & $0,74 \%$ \\
Lee S & 10 & $0,74 \%$ \\
Yang X & 9 & $0,67 \%$ \\
Denny JC & 8 & $0,59 \%$ \\
Li Q & 8 & $0,59 \%$ \\
Li Y & 8 & $0,59 \%$ \\
\hline
\end{tabular}

Fonte: Os autores (2019).

Analisando-se a Tabela 1, nota-se que o autor com a maior quantidade de registros produziu 12 artigos, concentrando $0,89 \%$ da produção total. Observa-se, também, que a produção destes dez autores listados 
corresponde a 7,16\% do total já produzido até o momento. Aplicando-se a lei de Lotka, verifica-se que de fato é possível identificar uma relação inversamente proporcional entre a quantidade de autores e artigos produzidos ${ }^{11,13,17}$. Essa relação é demonstrada no Gráfico 2.

\section{Gráfico 2 - Relação quantidade de artigos por autor}

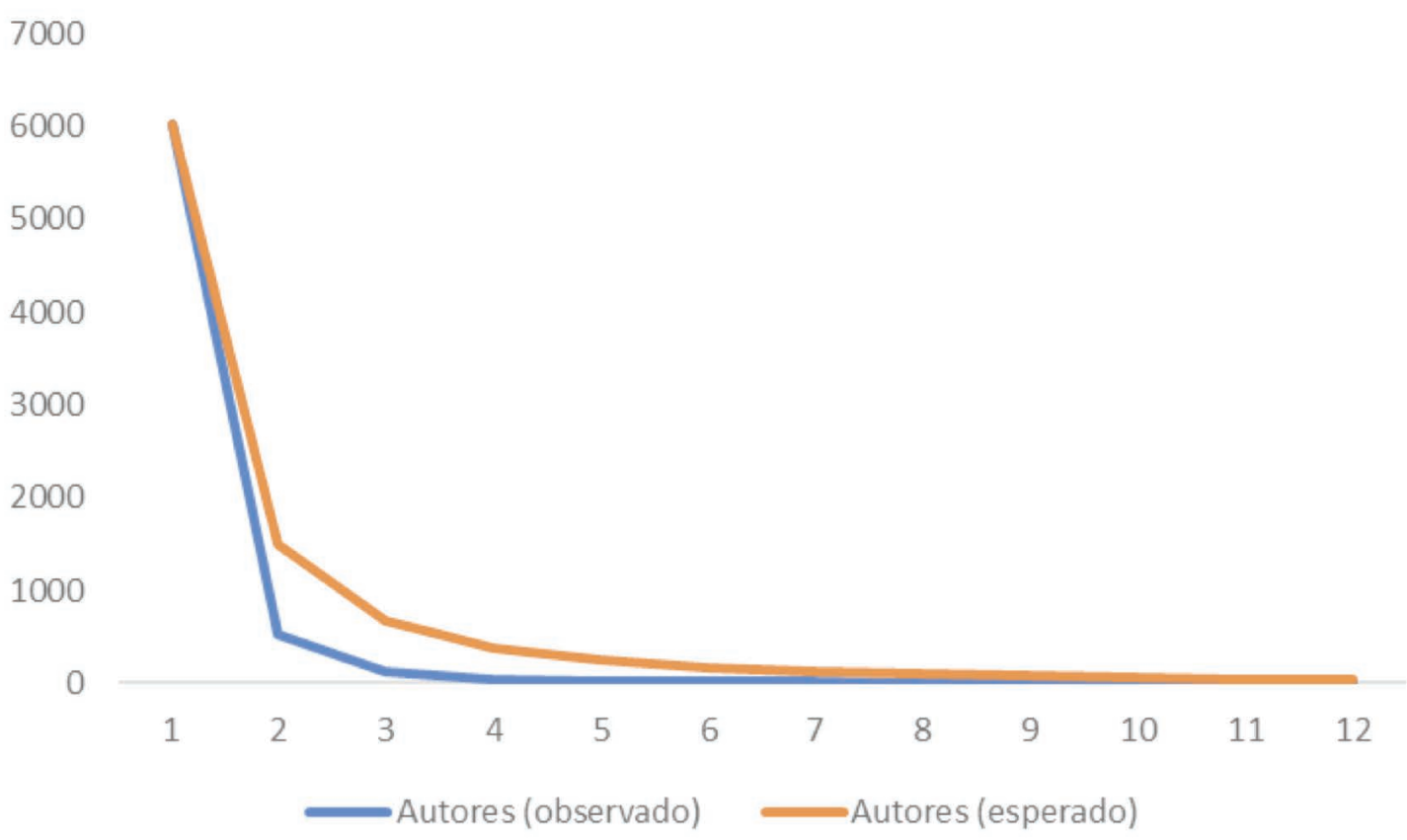

Fonte: Os autores (2019).

Ao se analisar o Gráfico 2, percebe-se uma curva em formato ' $J$ invertido' que representa o pensamento 'muitos com pouco e poucos com muito', uma vez que, quanto maior o número de artigos produzidos, menor é a quantidade de autores ${ }^{11,13,17}$. Nesse cenário, para o tema deste estudo, constatou-se que 6.020 autores (89,25\% do seu total) produziram apenas um artigo, enquanto a maior quantidade - 12 artigos foi produzida apenas por um autor. No tocante ao impacto dos autores, utilizou-se o total de citações e o índice-h como métricas de avaliação. O resultado dos dez autores com o maior impacto é listado na Tabela 2.

Tabela 2 - Autores com maior impacto

\begin{tabular}{lcclcc}
\multicolumn{1}{c}{ Autor } & Índice-h & $\begin{array}{c}\text { Início da } \\
\text { produção }\end{array}$ & \multicolumn{1}{c}{ Autor } & Total de citações & $\begin{array}{c}\text { Início da } \\
\text { produção }\end{array}$ \\
Cook DJ & 7 & 2009 & Cook DJ & 437 & 2009 \\
Li Q & 7 & 2011 & Schmitter Edgecombe M & 384 & 2011 \\
Kim J & 6 & 2011 & Hossain M & 358 & 2011 \\
An L & 6 & 2014 & Islam SMR & 356 & 2014 \\
Im J & 6 & 2011 & Kabir MH & 356 & 2011 \\
Ng A & 6 & 2013 & Kwak D & 356 & 2013 \\
Ng B & 6 & 2014 & Kwak KS & 356 & 2014 \\
Ng K & 6 & 2012 & Davatzikos C & 288 & 2012 \\
Lee S & 5 & 2013 & Lee S & 283 & 2013 \\
Denny JC & 5 & 2013 & Yang G & 264 & 2013 \\
\hline
\end{tabular}

Fonte: Os autores (2019). 
Conforme exposto na Tabela 2, o autor Cook DJ é o autor com o maior impacto em matéria de citação, considerando que o total de vezes que ele foi citado chegou a 437. Com relação ao índice-h, o mesmo autor divide com o pesquisador Li Q a primeira posição, ambos apresentando um índice-h igual a sete (7), o que significa que, ao ranquear as publicações desses autores em ordem decrescente de citação, verifica-se que apresentam sete artigos com, pelo menos, sete citações ${ }^{17}$.

Considerando os países dos autores, os cinco com o maior número de artigos são, respectivamente: a) Estados Unidos, com 35,55\% (481/1.353) dos artigos produzidos; b) Reino Unido, com 9,16\% (124/1.353) da produção analisada; c) China, cuja produção concentra 8,43\% (114/1.353) do total; d) Coreia, com 71 artigos, que representam 5,25\% do total; e e) Espanha, com 5,10\% (69/1.353) da produção total. Por sua vez, o Brasil apresenta 15 artigos (1,10\%).

Quanto aos artigos mais citados, verificou-se que dos dez primeiros, quatro foram produzidos no ano de 2015, dois no ano de 2011, dois em 2009 e, cada um dos anos de 2014 e 2016 contou com um artigo. A relação desses dez é apresentada na Tabela 3.

Tabela 3 - Artigos mais citados

\begin{tabular}{|c|c|c|c|}
\hline Artigo & Ano & $\begin{array}{l}\text { Total de } \\
\text { citações }\end{array}$ & $\begin{array}{l}\text { Citações } \\
\text { por ano }\end{array}$ \\
\hline The internet of things for health care: a comprehensive survey ${ }^{32}$ & 2015 & 356 & 89,00 \\
\hline $\begin{array}{l}\text { Classification of brain tumor type and grade using MRI texture and } \\
\text { shape in a machine learning scheme }{ }^{33}\end{array}$ & 2009 & 222 & 22,20 \\
\hline $\begin{array}{l}\text { Harnessing context sensing to develop a mobile intervention for } \\
\text { depression }^{34}\end{array}$ & 2011 & 190 & 23,75 \\
\hline $\begin{array}{l}\text { Discovering activities to recognize and track in a smart } \\
\text { environment }{ }^{35}\end{array}$ & 2011 & 186 & 23,25 \\
\hline Machine learning in medicine $e^{36}$ & 2015 & 146 & 36,50 \\
\hline $\begin{array}{l}\text { Cellphone-based hand-held microplate reader for point-of-care } \\
\text { testing of enzyme-linked immunosorbent assays }{ }^{37}\end{array}$ & 2015 & 140 & 35,00 \\
\hline $\begin{array}{l}\text { Computer-based personality judgments are more accurate than } \\
\text { those made by humans }{ }^{38}\end{array}$ & 2015 & 140 & 35,00 \\
\hline $\begin{array}{l}\text { A health-IoT platform based on the integration of intelligent } \\
\text { packaging, unobtrusive bio-sensor, and intelligent medicine box }{ }^{39}\end{array}$ & 2014 & 140 & 28,00 \\
\hline $\begin{array}{l}\text { Migration and stopover in a small pelagic seabird, the Manx } \\
\text { shearwater Puffinus puffinus: insights from machine learning }\end{array}$ & 2009 & 125 & 12,50 \\
\hline $\begin{array}{l}\text { Deep patient: an unsupervised representation to predict the future } \\
\text { of patients from the electronic health records }{ }^{41}\end{array}$ & 2016 & 123 & 41,00 \\
\hline
\end{tabular}

Fonte: Os autores (2019).

Observa-se, na Tabela 3, que o artigo com maior número de citações ${ }^{33}$ recebeu um total de 356 , apresentando um média de 89,00 citações por ano. O segundo artigo mais citado ${ }^{33}$ apresenta 222, com uma média de 22,20 citações por ano. O que obteve o maior número de citações consiste em uma revisão da literatura, que analisa os avanços das tecnologias de assistência baseadas em IoT, com foco nos recursos de segurança e privacidade.

Os autores propõem um modelo de segurança para minimizar riscos de segurança. O segundo artigo com o maior número de citações investiga o uso de métodos de classificação no tocante ao reconhecimento de padrões em diferentes tipos de tumores cerebrais, contemplando as etapas de definição da região de interesse, da extração e seleção de características e, por fim, a classificação. Quanto às fontes de publicação desses materiais, verificou-se que há 498 fontes. Aplicando-se a lei de Bradford, identificou-se que, na 
primeira zona, correspondente aos periódicos mais devotos ao tema, encontram-se oito periódicos, responsáveis por 34,96\% da produção. Na segunda zona, há 102 periódicos, que concentram 32,08\% da produção e, por fim, na zona de número três, 388 periódicos concentram 32,96\% da quantidade publicada.

Isso demonstra que há aderência à lei de Bradford, pois cada zona equivale a $1 / 3$ da produção total ${ }^{11} \mathrm{e}$ um pequeno grupo de periódicos, representados pela zona 1, concentram a maior parte das publicações da área ${ }^{11,14,17}$. A síntese das considerações é apresentada na Tabela 4.

Tabela 4 - Zonas de periódicos identificadas

\begin{tabular}{cccc} 
Zona & Frequência & Frequência (\%) & Produção (\%) \\
\hline Zona 1 & 8 & $1,61 \%$ & $34,96 \%$ \\
Zona 2 & 102 & $20,48 \%$ & $32,08 \%$ \\
Zona 3 & 388 & $77,91 \%$ & $32,96 \%$ \\
\hline
\end{tabular}

Fonte: Os autores (2019).

Com relação à 'Zona 1', ou seja, os periódicos com mais artigos, está concentrado no IEEE Access o maior número, com 93 registros, seguido pela revista PLOS ONE, com um total de 90 artigos. Compõem esta zona, ainda, os seguintes periódicos: Sensors (88); Journal of Medical Internet Research (52); Scientific Reports (43); Journal of the American Medical Informatics Association (39); Journal of Biomedical Informatics (36) e BMC Medical Informatics and Decision Making. No tocante à colaboração entre países, as cinco principais parcerias consistem nas relações entre: a) Reino Unido e Estados Unidos (48); b) China e Estados Unidos (44); c) Estados Unidos e Canadá (25); d) Estados Unidos e Austrália (19); e e) Estados Unidos e Alemanha (18). Com relação às colaborações envolvendo o Brasil, as principais relações identificadas consistem em: a) Brasil e Portugal (6); b) Brasil e Israel (3); c) Canadá e Brasil (3); d) Irlanda e Brasil (3); e) Japão e Brasil (3); e f) Estados Unidos e Brasil (3).

Quanto ao país de origem dos autores, observou-se que os cinco mais citados foram: a) Estados Unidos, com 6.601 citações, média de 13,72 por ano; b) Reino Unido, com 1.390 citações, média de 11,21 por ano; c) Coreia, com 570 citações, apresentando uma média de 8,03 por ano; d) Espanha, com 500 citações, tendo uma média de 7,24 por ano; e e) Canadá, com 357 citações, com uma média de 7,28 por ano. O Brasil registra 30 citações, apresentando uma média de duas por ano. No que diz respeito às instituições mais produtivas - considerando os autores - as dez principais são elencadas na Tabela 5 .

Tabela 5 - Instituições mais produtivas

\begin{tabular}{lcc}
\hline Instituição & Quantidade de artigos & Quantidade de artigos (\%) \\
Stanford University (USA) & 62 & $4,58 \%$ \\
University of Oxford (Reino Unido) & 51 & $3,77 \%$ \\
Vanderbilt University (USA) & 49 & $3,62 \%$ \\
Harvard Medical School (USA) & 45 & $3,33 \%$ \\
University of Pittsburgh (USA) & 43 & $3,18 \%$ \\
University of California, Los Angeles (USA) & 42 & $3,10 \%$ \\
University California, San Diego (USA) & 40 & $2,96 \%$ \\
University of Michigan (USA) & 38 & $2,81 \%$ \\
Harvard University (USA) & 34 & $2,51 \%$ \\
University of California San Francisco (USA) & 34 & $2,51 \%$ \\
\hline
\end{tabular}

Fonte: Os autores (2019). 
Analisando-se a Tabela 5, percebe-se que das dez principais instituições, nove estão localizadas nos Estados Unidos, e a única exceção é a Universidade de Oxford, no Reino Unido. A Universidade de Stanford é a instituição com o maior número de artigos produzidos, sendo responsável por 4,58\% da produção até o momento.

Quanto à análise das palavras-chave definidas pelos autores, contabilizaram-se 3.427 palavras diferentes, das quais, as expressões machine learning (431) e internet of things (97) foram as mais empregadas. O número expressivo destas duas justifica-se por serem parte da estratégia de busca empregada.

Para aplicar-se a lei de Zipf e o ponto de transição de Goffman $\left({ }^{T}\right.$ ), foi necessário ordenar as 3.427 palavras-chave, considerando as respectivas frequências de ocorrência (" $f$ ”). Em seguida, elas foram classificadas em ordem decrescente de frequência de ocorrência para obter-se a ordem de série (" $r$ "). Por fim, multiplicou-se a ordem de série pela frequência de ocorrência, obtendo-se assim a constante da primeira lei de Zipf ( $C$ ) . O resultado destas etapas é apresentado na Tabela 6.

Tabela 6 - Frequência das palavras-chave (autores) para a primeira lei de Zipf

\begin{tabular}{cccc|cccc}
$\begin{array}{c}\text { Quanti- } \\
\text { dade }\end{array}$ & $\begin{array}{c}\text { Ordem de } \\
\text { série "r" }\end{array}$ & $\begin{array}{c}\text { Frequência } \\
\text { "f" }\end{array}$ & $\begin{array}{c}\text { Constante } \\
(\mathbf{r * f =} \mathbf{f})\end{array}$ & $\begin{array}{c}\text { Quanti- } \\
\text { dade }\end{array}$ & $\begin{array}{c}\text { Ordem de } \\
\text { série "r" }\end{array}$ & $\begin{array}{c}\text { Frequência } \\
\text { "f" }\end{array}$ & $\begin{array}{c}\text { Constante } \\
(\mathbf{r} * \mathbf{f}=\mathbf{c})\end{array}$ \\
\hline 1 & 1 & 54 & 54 & 1 & 15 & 14 & 210 \\
1 & 2 & 51 & 102 & 4 & 16 & 13 & 208 \\
1 & 3 & 49 & 147 & 1 & 17 & 12 & 204 \\
1 & 4 & 45 & 180 & 8 & 18 & 11 & 198 \\
1 & 5 & 43 & 215 & 10 & 19 & 10 & 190 \\
1 & 6 & 35 & 210 & 8 & 20 & 9 & 180 \\
1 & 7 & 30 & 210 & 9 & 21 & 8 & 168 \\
1 & 8 & 29 & 232 & 13 & 22 & 7 & 154 \\
1 & 9 & 26 & 234 & 16 & 23 & 6 & 138 \\
1 & 10 & 25 & 250 & 38 & 24 & 5 & 120 \\
1 & 11 & 24 & 264 & 53 & 25 & 4 & 100 \\
3 & 12 & 17 & 204 & 114 & 26 & 3 & 78 \\
2 & 13 & 16 & 208 & 298 & 27 & 2 & 54 \\
2 & 14 & 15 & 210 & 2834 & 28 & 1 & 28 \\
\hline
\end{tabular}

Fonte: Os autores (2019).

Tendo em vista que as palavras-chave mais citadas corresponderam aos termos de busca empregados, optou-se por retirá-las para o cálculo da lei de Zipf. Sendo assim, as principais palavras-chave utilizadas foram: a) data mining (54); b) electronic health records (51); c) natural language processing (49); d) big data (45); e) deep learning (43); f) classification (30); e f) artificial intelligence (29). Em termos gráficos, os resultados elencados na Tabela 6 podem ser analisados no Gráfico 3. 
Gráfico 3-Aplicação da primeira lei de Zipf

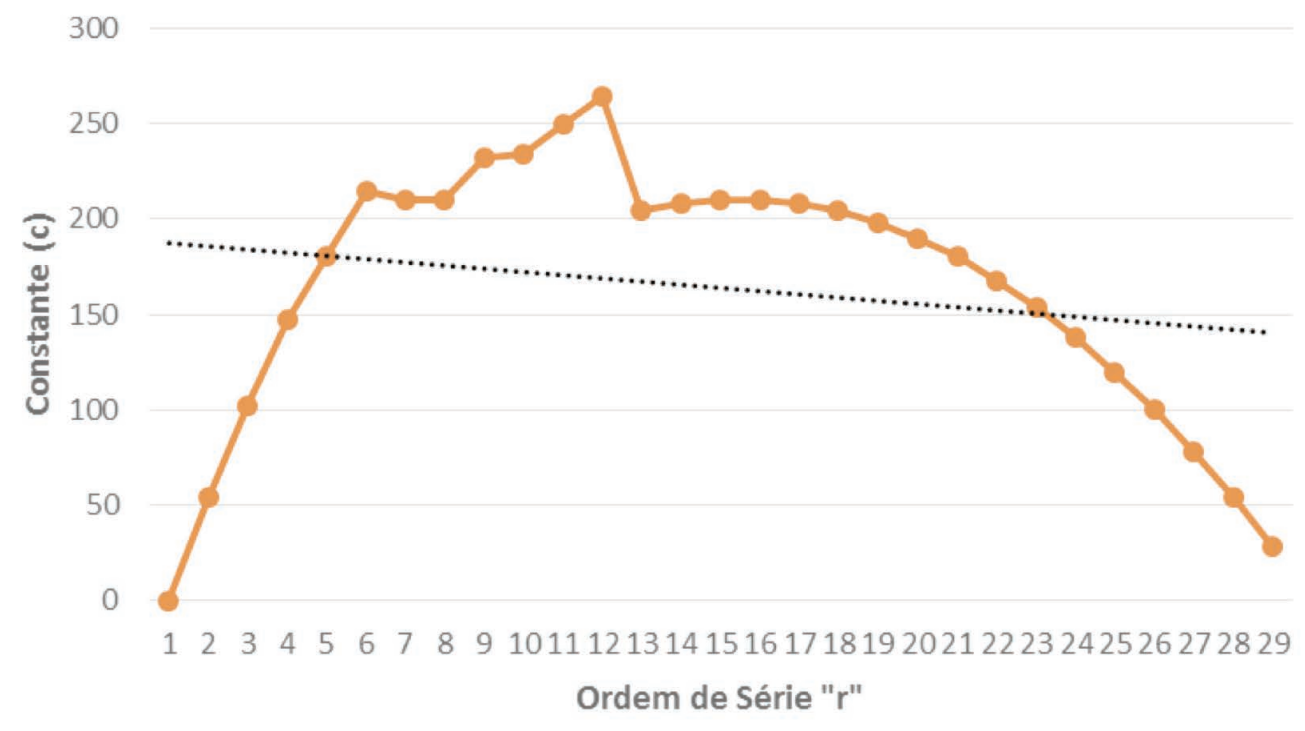

Fonte: Os autores (2019).

Observando-se o Gráfico 3, tem-se que o ponto máximo corresponde a 264 - na ordem de série 11. À esquerda deste ponto encontram-se as palavras de maior ocorrência (frequência), enquanto, à direita, localizam-se as palavras-chave com menor ocorrência. Tendo o resultado para a primeira lei de Zipf, calculou-se o ponto de transição de Goffman $\left({ }^{T}\right.$ ), representado pela equação 5 .

$$
n=-1 \sqrt{\frac{1+8(2834)}{2}}=n=74,79
$$

Ao se arredondar o valor $n$ obtido na equação 5 , tem-se que o ponto ${ }^{T}$ corresponde à $75^{\text {a }}$ palavra, que é a expressão 'diabetes mellitus', com frequência igual a seis. Desta forma, consideram-se todas as palavras com a mesma frequência desta expressão como as mais significativas, sendo elas as seguintes expressões: a) artificial neural networks; b) decision support systems; c) diagnosis; d) edge computing; e) epidemiology; f) epilepsy; g) faulth) diagnosis; i) health care; j) health informatics; k) heart failure; l) hypertension; m) influenza; $\mathrm{n}$ ) metagenomics; o) precision medicine; e p) smart home.

Buscando encontrar possíveis relações entre esses termos, elaborou-se uma rede de coocorrência por meio dos algoritmos de normalização ‘association' e agrupamento 'Walktrap'. A rede de coocorrência com as principais palavras-chave identificadas é demonstrada na Figura 1. 
social media

natural language processing

electronic health records

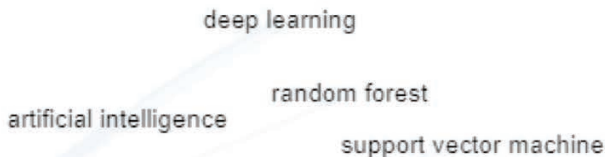

\section{machine learning}

prediction

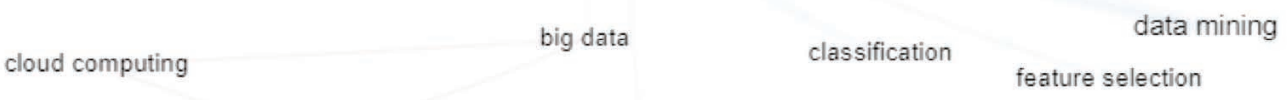

internet of things

ambient assisted living

wearable sensors

iot

e-health

Figura 1 - Rede de coocorrência para as palavras-chave (autores)

Fonte: Os autores (2019).

Identificaram-se tópicos interessantes para a realização de novos estudos na área da saúde, como as seguintes expressões relacionadas: internet das coisas, ambientes de vida assistida (ambient assisted living), sensores vestíveis (wearable sensors) e computação em nuvem (cloud computing). Também se atenta para o termo 'e-health', indicando que a saúde caminha cada vez mais para o formato digital, no qual diversas ferramentas podem ser utilizadas em conjunto.

Os termos relacionados ao aprendizado de máquina indicam uma possível exploração de técnicas de processamento delinguagem natural (natural language processing) em registros eletrônicos de saúde (electronic health records) e mídia social (social media). Além disso, notam-se técnicas relacionadas à inteligência artificial (artificial intelligence), como o uso de deep learning e a aplicação de heurísticas de mineração de dados (data mining), como support vector machine e random forest em tarefas de predição e classificação.

Em seguida, analisaram-se as Keywords Plus - um tipo de vocabulário controlado definido pela Web of Science - e identificou-se que os principais tópicos foram: a) classification (135); b) health (118); c) system (81); d) care (68); e) disease (58); f) risk (58); f) internet (57); g) prediction (52); h) health care (50); e diagnosis (45). Buscando encontrar possíveis relações entre estes termos, elaborou-se uma rede de coocorrência por meio dos algoritmos de normalização 'association' e agrupamento ‘Walktrap'. A rede de coocorrência para as principais keyword plus identificadas é demonstrada na Figura 2. 


\section{regression \\ model \\ association \\ prediction \\ classification}

identification

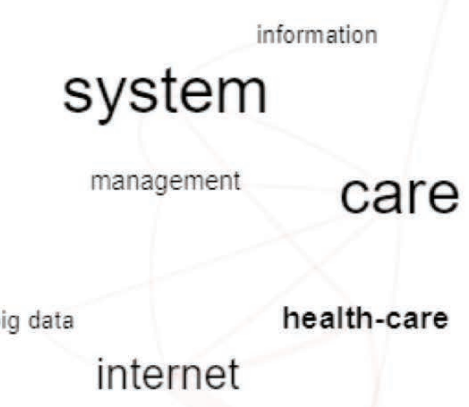

health

disease risk

prevalence diagnosis

Figura 2 - Rede de coocorrência para as Keywords Plus

Fonte: Elaborada pelos autores (2019).

Com base na Figura 2, observam-se novos tópicos que podem servir como objeto de estudo, tais como o uso da tecnologia como possível instrumento de predição, identificação e diagnóstico. Percebem-se termos como mortalidade, doença e fatores de risco, indicando possíveis abordagens para o emprego do aprendizado de máquina, nas quais as tarefas de classificação, associação e regressão podem ser valiosas ferramentas.

Por fim, verifica-se a presença de uma relação entre big data e sistemas de gestão da informação e cuidados da saúde, o que se demonstra como um campo interessante para o desenvolvimento de novos sistemas de apoio à tomada de decisão na área da saúde.

\section{Considerações finais}

O objetivo deste artigo é analisar a produção científica do período de 2009 a 2019 a respeito da internet das coisas e do aprendizado de máquina na área da saúde por meio da bibliometria. Com este estudo, verificou-se a necessidade da área da saúde integrar as tecnologias desenvolvidas e fornecidas pela internet das coisas e pelo aprendizado de máquina.

Isso se deve ao fato de o atual contexto ser caracterizado pelo alto volume de dados e uma população cada vez mais envelhecida e dependente de cuidados fora dos ambientes hospitalares, o que demanda alterações quanto ao uso das tecnologias da informação e comunicação para garantir o seu bem-estar.

Em virtude da internet das coisas, os procedimentos da área da saúde tendem a ser mais ágeis, posto que em ambientes de vida assistida, por exemplo, há possibilidade de que sistemas baseados em sensores wireless enviem dados diretamente para sistemas com os quais os profissionais de saúde consigam interagir. Para isso, as aplicações em nuvem (cloud computing) surgem como grandes alicerces para novos desenvolvimentos.

Identificou-se que tópicos como registros eletrônicos de saúde (electronic health records) e mídias sociais podem ser explorados por meio de processamento de linguagem natural (natural language processing). 
Algoritmos e estratégias de aprendizado de máquina como deep learning, random forest e support vector machine apresentaram-se como tópicos já empregados na área da saúde e, por isso, podem ser aplicados às necessidades da área e dos profissionais, tornando-se ferramentas interessantes para o desenvolvimento de novos produtos e serviços.

Quanto à área de pesquisa, há um crescimento no volume da produção científica ao longo do período, cuja tendência é aumentar ao longo dos anos, tendo em vista que até o momento, o ano de 2019 totaliza 128 registros, que correspondem a 9,46\% do volume produzido.

Os Estados Unidos são o principal polo de pesquisa, sendo o país com o maior número de autores, o que mais recebeu citações, o detentor das principais relações de colaboração entre países e o local que abriga nove das dez principais instituições de pesquisa.

O Brasil, por sua vez, colabora com o tema, contudo, com apenas 15 trabalhos associados ao país, que receberam 30 citações. Em termos de colaboração, o Brasil já estabeleceu parcerias com países como Portugal e Israel e países como Canadá, Irlanda, Japão e Estados Unidos têm colaborado com o país em termos de produção.

Por fim, incentivam-se novas pesquisas que explorem algumas abordagens identificadas ao longo deste artigo, por meio de estudos de caso que possam demonstrar que a internet das coisas, assim como o aprendizado de máquina são tecnologias que irão agregar valor aos procedimentos, aos profissionais e à área de saúde.

\section{Referências}

1. Ara A, Ara A. Case study: integrating IoT, streaming analytics and machine learning to improve intelligent diabetes management system [Internet]. In: International Conference on Energy, Communication, Data Analytics and Soft Computing; 2017 Aug 1-2; Chennai, India. New Jersey: IEEE; 2017 [cited 2018 Aug 19]. p.3179-82. Available from: https://ieeexplore.ieee.org/document/8390043/

2. Costa CA, Pasluosta CF, Eskofier B, Silva DB, Righia RR. Internet of health things: toward intelligent vital signs monitoring in hospital wards. Artifi Intell Med [Internet]. 2018 [cited 2018 Aug 19];89:61-9. Available from: https://www.sciencedirect.com/science/article/pii/S0933365717301367

3. Sianaki OA, Yousefi A, Tabesh AR, Mahdavi M. Internet of everything and machine learning applications: issues and challenges [Internet]. In: 32nd International Conference on Advanced Information Networking and Applications Workshops; 2018 May 16-18; Krakow, Poland. New Jersey: IEEE; 2018 [cited 2018 Aug 19]. p. 704-8. Available from: https://ieeexplore.ieee.org/document/8418156/

4. Sundaravadivel P, Kougianos E, Mohanty SP, Ganapathiraju MK. Everything you wanted to know about smart health care: evaluating the different technologies and components of the internet of things for better health. IEEE Consum Elect Mag [Internet]. 2018 Jan [cited 2018 Aug 19]; 7(1):18-28. Available from: https://ieeexplore.ieee.org/document/8197457/

5. Pulavskyi AA, Krivenko SS, Kryvenko LS. Functional diagnostic using electrical impedance tomography reconstruction and the internet of things [Internet]. In: 6th Mediterranean Conference on Embedded Computing; 2017 June 11-15; Bar, Montenegro. New Jersey: IEEE; 2017 [cited 2018 Aug 19]. Available from: https://ieeexplore.ieee.org/document/7977241/

6. Zhu N, Diethe T, Camplani M, Tao L, Burrows A, Twomey N, et al. Bridging e-health and the internet of things: the SPHERE project. IEEE Int Syst [Internet]. 2015 July-Aug [cited 2018 Aug 19]; 30(4):39-45. Available from: https://ieeexplore.ieee.org/document/7156004/

7. Nithya B, Ilango V. Predictive analytics in health care using machine learning tools and techniques [Internet]. In: International Conference on Intelligent Computing and Control Systems; 2017 June 1516; Madurai, India. New Jersey: IEEE; 2017 [cited 2018 Aug 19]. p. 492-9. Available from: https:// ieeexplore-ieee-org.ez22.periodicos.capes.gov.br/document/8250771/

8. Pimenta $A A$, Portela ARMR, Oliveira CB, Ribeiro RM. A bibliometria nas pesquisas acadêmicas. Scientia [Internet] 2017 [citado em 2019 out. 26];4(7):1-13. Disponível em: https://bit.ly/2Vqv]25

9. Mugnaini R, Jannuzzi PM, Quoniam L. Indicadores bibliométricos da produção científica brasileira: uma análise a partir da base Pascal. Ci Inf [Internet]. 2004 jul.-ago. [citado em 2018 ago. 19];33(2):123-31. Disponível em: http://www.scielo.br/pdf/ci/v33n2/a13v33n2 
10. Aria M, Cuccurullo C. Bibliometrix: An R-tool for comprehensive science mapping analysis. J Informetr [Internet]. 2017 [cited 2019 Apr 26];11(4):959-75. Available from: https://www.sciencedirect.com/ science/article/abs/pii/S1751157717300500

11. Araújo C. Bibliometria: evolução histórica e questões atuais. Em Questão [Internet]. 2006 [citado em 2019 abr. 26];12(1):11-32. Disponível em: https://dialnet.unirioja.es/servlet/articulo?codigo=6134719

12. Parra M, Coutinho R, Pessano E. Um breve olhar sobre a cienciometria: origem, evolução, tendências e sua contribuição para o ensino de ciências. Rev Contexto Educ [Internet] 2019 [citado em 2019 abr. 27];34(107):126-41. Disponível em: https://revistas.unijui.edu.br/index.php/contextoeducacao/article/ view/7267

13. Lotka A. The frequency distribution of scientific productivity. J Washington Acad Sci [Internet]. 1926 [cited 2019 Apr 26];16(12):317-23. Available from: https://www.jstor.org/ stable/24529203?seq $=1$ \#page scan tab contents

14. Bradford S. Sources of information on specific subjects. Engineering. 1934;137:85-6.

15. Zipf G. Human behavior and the principle of least effort. New York: Hafner; 1972.

16. Mattedi, MA, Spiess, MR. A avaliação da produtividade científica. His Ciên Saúde-Manguinhos. [Internet] 2017 [citado em 2019 abr. 27];24(3):623-43. Disponível em: http://www.redalyc.org/ pdf/3861/386153003005.pdf

17. Andrés A. Measuring academic research. Oxford: Chandos; 2009.

18. Vanti, NAP. Da bibliometria à webometria: uma exploração conceitual dos mecanismos utilizados para medir o registro da informação e a difusão do conhecimento. Ci Inf [Internet] 2002 [citado em 2019 abr. 27];31(2):152-62. Disponível em: http://www.scielo.br/pdf/ci/v31n2/12918

19. Cassettari R, Pinto A, Rodrigues R, Santos L. Comparação da Lei de Zipf em conteúdos textuais e discursos orais. Prof Inform [Internet]. 2015 [citado em 2019 maio 15];24(2):157. Disponível em: https://www.researchgate.net/publication/277930184 Comparacao da Lei de Zipf em conteudos textuais e discursos orais

20. Santos R, Kobashi N. Bibliometria, cientometria, infometria: conceitos e aplicações. Pesq bras Ci Inf [Internet]. 2009 [citado em 2019 maio 15];2(1):155-72. Disponível em: https://repositorio.ufpe. br/bitstream/123456789/10089/1/BIBLIOMETRIA\%2c\%20CIENTOMETRIA\%2c\%20INFOMETRIA CONCEITOS\%20E\%20APLICA\%C3\%87\%C3\%95ES.pdf

21. Guedes V. A bibliometria e a gestão da informação e do conhecimento científico e tecnológico: uma revisão da literatura. Ponto Acesso [Internet]. 2012 [citado em 2019 maio 15];6(2):74-109. Disponível em: https://rigs.ufba.br/index.php/revistaici/article/view/5695/4591

22. Soteriades E, Falagas M. A bibliometric analysis in the fields of preventive medicine, occupational and environmental medicine, epidemiology, and public health. BMC Public Health. 2006 [cited 2019 Apr 27];6(1). Available from: https://bmcpublichealth.biomedcentral.com/articles/10.1186/1471-2458-6-301

23. Santin, DM, Nunez, ZAG, Moura, AMM. Produção científica brasileira sobre células-tronco de 2000 a 2013: características e colaboração internacional. Rev Eletron Comum Inf Inov Saude [Internet] 2015 [citado 26 abr. 2019];9(2):1-16. Disponível em: https://www.reciis.icict.fiocruz.br/index.php/reciis/ article/view/965/pdf 339

24. Skvoretz J, Dyer K, Daley E, Debate R, Vamos C, Kline N et al. Research and practice communications between oral health providers and prenatal health providers: a bibliometric analysis. Matern Child Health J [Internet]. 2016 [cited 2019 Apr 26];20(8):1607-19. Available from: https://link.springer.com/ article/10.1007/s10995-016-1960-x

25. Martins MFM. Análise bibliométrica de artigos científicos sobre o vírus Zika. Rev Eletron Comum Inf Inov Saude [Internet] 2016 [citado 27 abr. 2019];10(1): 1-9. Disponível em: https://www.reciis.icict.fiocruz. br/index.php/reciis/article/view/1096/pdf 1096

26. Areias F, Abrão F, Moura L, Silva R, Costa S, Silva C. Análise bibliométrica de artigos sobre laringectomia total. Rev Baiana Enferm [Internet]. 2016 [citado 26 abr. 2019];30(3):1-11. Disponível em: https:// portalseer.ufba.br/index.php/enfermagem/article/view/15790

27. Alencar MSM, Bochner R, Dias MFF, Antunes AMS. Análise da produção científica brasileira sobre nanotecnologia e saúde. Rev Eletron Comum Inf Inov Saude [Internet] 2017 [citado 2019 abr. 26];11(1):1-16. Disponível em: https://www.reciis.icict.fiocruz.br/index.php/reciis/article/ view/1199/2094 
28. Sweileh WM, Al-jabi SW, Abutaha AS, Zyoud SH, Anayah FMA, Sawalha A. Bibliometric analysis of worldwide scientific literature in mobile-health: 2006-2016. BMC Med inform Decis Mak [Internet] 2017 [cited 2019 Apr 26];17(1):1-12. Available from: https://bmcmedinformdecismak.biomedcentral.com/ articles/10.1186/s12911-017-0476-7

29. Fernandes MRCC, Silva CRL, Souza JHS, Lima LCM, Santos ICRV, França I. Produtividade científica acerca da neoplasia maligna bucal e da reabilitação bucal: uma análise bibliométrica. Cogitare Enferm [Internet] 2017 [citado em 2019 abr. 26];22(4). Disponível em: http://www.redalyc.org/articulo. oa?id $=483654880004$

30. Sweileh, WM. A bibliometric analysis of health-related literature on natural disasters from 1900 to 2017. Health Res Policy Syst [Internet] 2019 [cited 2019 Apr 26];17(1):1-11.Available from: https://healthpolicy-systems.biomedcentral.com/articles/10.1186/s12961-019-0418-1

31. Tran B, Vu G, Ha G, Vuong Q, Ho M, Vuong T, et al. Global evolution of research in artificial intelligence in health and medicine: a bibliometric study. ] Clin Med [Internet] 2019 [cited 2019 Apr 26];8(3). Available from: https://www.mdpi.com/2077-0383/8/3/360/htm

32. The Internet of things for health care: a comprehensive survey. IEEE Access [Internet]. 2015 [cited 2019 May 15]; (3):678-708. Available from: https://ieeexplore.ieee.org/stamp/stamp. jsp?tp=\&arnumber $=7113786$

33. Zacharaki E, Wang S, Chawla S, Soo Yoo D, Wolf R, Melhem E, et al. Classification of brain tumor type and grade using MRI texture and shape in a machine learning scheme. Magn Reson Med [Internet]. 2009 [cited 2019 May 15]; 62(6):1609-18. Available from: https://www.ncbi.nlm.nih.gov/ pubmed/19859947

34. Burns M, Begale M, Duffecy J, Gergle D, Karr C, Giangrande E. et al. Harnessing context sensing to develop a mobile intervention for depression. J Med Internet Res [Internet]. 2011 [cited 2019 May 15];13(3): e55. Available from: https://www.jmir.org/2011/3/e55/

35. Rashidi $P$, Cook D, Holder L, Schmitter-Edgecombe M. Discovering activities to recognize and track in a smart environment. IEEE T Knowl Data En [Internet]. 2011 [cited 2019 May 15]; 23(4):527-39. Available from: https://ieeexplore.ieee.org/abstract/document/5560652

36. Rajkomar A, Dean J, Kohane I. Machine learning in medicine. N Engl J Med [Internet]. 2019 [cited 2019 May 15];380(14):1347-58. Available from: https://www.nejm.org/doi/full/10.1056/NEJMra1814259

37. Berg B, Cortazar B, Tseng D, Ozkan H, Feng S, Wei Q et al. Cellphone-based hand-held microplate reader for point-of-care testing of enzyme-linked immunosorbent assays. ACS Nano [Internet]. 2015 [cited 2019 May 15];9(8):7857-66. Available from: https://www.ncbi.nlm.nih.gov/pubmed/26159546

38. Youyou W, Kosinski M, Stillwell D. Computer-based personality judgments are more accurate than those made by humans. Pro Natl Acad Sci [Internet]. 2015 [cited 2019 May 15];112(4):1036-40. Available from: https://www.pnas.org/content/112/4/1036

39. Yang G, Xie L, Mantysalo M, Zhou X, Pang Z, Xu L et al. A health-iot platform based on the integration of intelligent packaging, unobtrusive bio-sensor, and intelligent medicine box. IEEE T Ind Inform [Internet]. 2014 [cited 2019 May 15];10(4):2180-91. Available from: https://ieeexplore.ieee.org/ document/6747344

40. Guilford T, Meade J, Willis J, Phillips R, Boyle D, Roberts S, et al. Migration and stopover in a small pelagic seabird, the Manx shearwater Puffinus puffinus: insights from machine learning. Proc Biol Sci [Internet]. 2009 [cited 2019 May 15];276(1660):1215-23. Available from: https://royalsocietypublishing. org/doi/full/10.1098/rspb.2008.1577

41. Miotto R, Li L, Kidd B, Dudley J. Deep patient: an unsupervised representation to predict the future of patients from the electronic health records. Sci Rep [Internet]. 2016 [cited 2019 May 15]; 6(1). Available from: https://www.nature.com/articles/srep26094 